\title{
Interculturalidad y TIC en los posgrados de educación en el Ecuador
}

\author{
Interculturality and TIC in postgraduate education proposals in \\ Ecuador
}

\author{
Fanny Tubay Zambrano \\ Universidad de Cuenca, Ecuador
}

\begin{abstract}
Resumen
La investigación ha destacado la necesidad de abordar la compleja realidad de las sociedades culturalmente diversas, y la importancia que para ello tiene entrelazar multiplicidad de saberes y necesidades de enseñanza-aprendizaje. Ante este reto, los posgrados en educación constituyen un ámbito de gran relevancia donde poner en valor una perspectiva interdisciplinar, en especial relacionando el enfoque intercultural y las tecnologías educativas. El presente estudio analiza 18 programas de posgrado en Ecuador con el fin de visibilizar en qué medida responden a este planteamiento. La investigación, de corte cualitativa, por un lado, selecciona los posgrados avalados por el órgano rector de la educación superior en el país y en vigencia. Y por otro, acude al análisis documental para revisar presupuestos teóricos en contraste con los diseños de la oferta seleccionada. Los resultados sugieren que en su mayoría la oferta de posgrados integra elementos para promover un diálogo de respeto, equidad y justicia social, en lo que podríamos considerar un cambio de paradigma. A pesar de ello, los resultados también visibilizan vacíos curriculares, que convierten lo intercultural y lo digital en meros elementos discursivos en lugar de componentes emergentes y transversales para la educación.
\end{abstract}

Palabras clave: Enfoque intercultural; Tecnologías educativas; Diversidad; Diálogo de saberes; Posgrado en educación
ঞ

\begin{abstract}
The research has highlighted the need to address the complex reality of culturally diverse societies, and importance of intertwining the multiplicity of knowledge and teaching-learning needs for this. Faced with this challenge, postgraduate courses in education constitute a highly relevant area where to value an interdisciplinary perspective, especially relating the intercultural approach and educational technologies. This study analyzes 18 postgraduate programs in Ecuador in order to make visible to what extent they respond to this approach. The qualitative research, on the one hand, selects postgraduate courses endorsed by the governing body of higher education in the country and in force. And on the other, it goes to the documentary analysis to review theoretical budgets in contrast to the designs of the selected offer. Results suggest that most of the postgraduate offer includes elements to promote a dialogue of respect, equity and social justice, in what we could consider a paradigm shift. Despite this, the results also reveal curricular gaps, which turn the intercultural and the digital into mere discursive elements instead of emerging and transversal components for education.
\end{abstract}

Keywords: Intercultural approach; Educational technologies; Diversity; Dialogue of knowledge; Postgraduate education 


\section{INTRODUCCIÓN}

El trabajo explora la dimensión intercultural y de las tecnologías de la información y comunicación (en adelante TIC) en la oferta de posgrado en el Ecuador desde un análisis de los registros de programas ofertados y validados por el Consejo de Educación Superior (a partir de ahora CES) y desde la perspectiva de los estudios interculturales. Para Sánchez (2020) los estudios bajo esa mirada representan un espacio social, académico y emancipado donde convergen diversas voces, visiones y escenarios. En esas arenas, la educación intercultural y las TIC tienen gran incidencia, pues permiten el desarrollo de actividades artísticas, socio comunitarias, prácticas decoloniales y socioeducativas, todas capaces de promover experiencias educativas centradas en la integración social, la inclusión y el intercambio de saberes.

En la praxis podríamos estimar que tanto la educación pública como la democrática bien podrían representar ese espacio de convivencia armónica, desde donde se puede garantizar la atención a la diversidad y el dialogo intercultural (UNESCO, 2013), no obstante, su propuesta como modelo no es suficiente, pues aún hacen falta más sitios y sistemas educativos en los que se puedan cultivar (impartir, estudiar y someter a escrutinio y debate público) las cosmovisiones, sabidurías, tecnologías, los valores y principios, los modelos productivos y económicos, y las prácticas culturales de las propias comunidades (Mato, 2009).

De ahí que, existe aún existe la demanda de una oferta educativa que aloje nuevas dimensiones, visiones y formas diferentes para hacer lecturas más dinámicas y flexibles en los imaginarios sociales. En este contexto, la perspectiva intercultural y la integración de las TIC pueden contribuir a desarrollar una pedagogía liberadora (Freire, 1998), construida desde la ética y la cultura de mutuo respeto en contextos heterogéneos.

Derivado de este marco percibimos que la relación interculturalidad-tecnologías no es inusual, porque lo intercultural está ampliamente conectado e incorporado en internet desde múltiples perspectivas, formas e idiomas (Hepp, 2009). A tales efectos, la necesidad de trabajar desde un enfoque social transversal, sea presencial o por medio de la "digiculturalización" (la integración de lo cultural y lo virtual; Leiva \& Moreno, 2011) es impostergable si se quieren desarrollar competencias interculturales que provean de herramientas necesarias para competir en los campos laborales y comunicarse más allá de sus fronteras (Dietz, 2017).

La literatura científica evidencia también una relación visible entre las TIC y los estudios interculturales, ya sea en concomitancia con contextos diversos 
(minorías, culturas juveniles, género o multiculturalidad) o con programas curriculares y las habilidades que estos permiten incrementar desde una mirada que agrupa diversos campos y disciplinas.

\section{La interculturalidad: un término y una práctica en construcción}

La teoría alrededor de la educación intercultural sostiene que se trata de un término complejo y polisémico referido a las relaciones que existen dentro de la sociedad entre diversas constelaciones de mayoría-minoría, y que se definen en materia de cultura, etnicidad, lengua, denominación religiosa y/o nacionalidad (Dietz, 2017). Visto desde el campo de la educación, ese término también define las prácticas culturales y las formas en las que las personas aprenden y enseñan, y en la utilidad que tienen los saberes acorde a sus necesidades contextualizadas. De acuerdo con Fornet-Betancourt (2001) lo intercultural se ve en el reconocimiento real de cada cultura, pero también de cada grupo que aprende, alcanzando por tanto una visión del mundo que tiene algo que decir a todos.

Para hablar de lo intercultural como propuesta de transformación educativa en la educación superior, Walsh (2005) propone abordar el concepto desde una dimensión amplia que integra diferentes voces, visiones y saberes, involucrando además a todos los actores en los procesos e intereses comunes. Situándose en el contexto de Perú, un país sumamente diverso y pluricultural, la autora considera que la interculturalidad

\footnotetext{
tiene el rol crítico, central y prospectivo (no sólo en la educación, sino en todas las instituciones de la sociedad) de reconstruir, paso a paso, sociedades, sistemas y procesos educativos, sociales, políticos y jurídicos; y de accionar entre todos los peruanos indígenas, blancos, mestizos, cholos, negros, mulatos, asiáticos, árabes, etc. relaciones, actitudes, valores, prácticas, saberes y conocimientos fundamentados en el respeto e igualdad, el reconocimiento de las diferencias y la convivencia democrática (p.5).
}

Planteada de esa forma, la interculturalidad propone el camino más apropiado para buscar estrategias comunes de vida para todos, rechazando la esencialidad y la creación de espacios segregados para determinados grupos por cuestiones identitarias o étnicas. Se trata de un enfoque y un proceso que impulsa cambios sociales desde diferentes ramas, pero con particular incidencia en el ámbito educativo.

Para Aguado \& Mata (2017) lo intercultural es una metáfora que permite comprender lo que las personas, piensan, dicen y hacen en relación con la diversidad cultural desde la asunción de un compromiso por la equidad y la justicia social. No se trata de una política pública que depende únicamente del estado nación, sino 
de un pacto individual como seres humanos con la capacidad de ejercer un modelo de ciudadanía basado en el respeto por la diferencia y en la valoración de la diversidad como una fuente de riqueza. Como sostiene Walsh (2005), la interculturalidad no va a venir hacia nosotros espontáneamente, sino que todos tenemos la responsabilidad de buscarla y construirla, ya sea como universidad, ciudadanía o grupo social.

Contrariamente, cabe mencionar que no todas las señales apuntan a la óptica intercultural como medio de cambio progresivo. En palabras de Tipa (2018), la implementación de la educación intercultural ha causado fuertes críticas tanto administrativas como prácticas, teóricas y metodológicas. Juntar saberes modernos con los tradicionales ha supuesto problemas para las universidades interculturales en países como México, por lo que aplicar sistemas rígidos y estándares validando saberes universales permitió que se flexibilicen los procesos, es decir se faciliten para quienes presentan dificultades o desventajas. Aunque eso ha dado lugar a que estos proyectos se malinterpreten y se consideren de baja calidad.

Otro cuestionamiento lo formulan Charle et al. (2007) para quienes la noción de transferencia intercultural a nivel educativo en Latinoamérica está impregnada de ideas exógenas que son integradas a las culturas de destino desde categorías esencialistas que colocan el privilegio en quienes ostentan el poder de la cultura dominante. Se trata por tanto de un modelo educativo eurocéntrico que sugiere una peligrosa confusión entre los conceptos cultura y nacionalidad, tan frecuentes en los estudios y en la educación intercultural (Adam, 2007; García \& Granados, 1999; Mateos \& Dietz, 2014). De ahí que resulte importante construir lo intercultural desde un proyecto político, social y educativo que proceda desde abajo y desde adentro (los movimientos sociales) para garantizar procesos multidireccionales.

\section{Las Tic en la educación intercultural}

El paradigma inclusivo, comprensivo e intercultural que ofrece la tecnología (computadora, telefonía o acceso a internet, entre otras), resulta una de las posibilidades más pertinentes para ayudar a las personas a mantenerse conectadas entre sí y con el mundo, y cabe reconocer que en el campo educativo como herramienta está muy presente en los procesos de enseñanza y aprendizaje. Algunos autores como Alderete \& Formicelle (2006) consideran incluso que forman parte inexcusable de la educación en todos sus niveles, sin embargo, desde una perspectiva global observamos que en ocasiones sí y en otras no, su presencia es indispensable. De ahí que la diversidad proponga diferentes tipos de posibilidades y mecanismos de aprendizaje, y las Tic representan solo uno de ellos. 
Las instituciones educativas tienen un papel cohesionador para enfrentar los problemas crónicos de la sociedad. Por ello, una de las necesidades emergentes que deben afrontar es reflexionar sobre el rol de las TIC y su alcance para superar distancias entre realidades socioculturales (Priegue \& Leiva, 2012), y visibilizar la importancia de la otredad (Zambrano \& Sant Ana, 2020) sin importar condiciones de clase social, etnia, género o temporalidad.

Autores como Priegue \& Leiva (2012) sostienen que las TIC y sus herramientas virtuales, además de impulsar el desarrollo de una sociedad digital, activan una cultura social de involucramiento. Motivan a los más jóvenes a participar en la generación de comunidades virtuales, promueven el conocimiento y reconocimiento de las diferencias culturales como claves positivas de enriquecimiento personal y social, facilitan la comunicación intercultural a través del contacto de personas y culturas diversas, y llegan a favorecer la participación activa y colaborativa. Por lo que se entiende que su ensamblaje epistémico es parte del proceso intercultural en una era globalizada, en la que las personas tienen diferentes motivaciones, desarrollan otras habilidades, exploran otras formas de relacionarse socioculturalmente, y a su vez eligen las modalidades que están a su alcance desde una función práctica.

Para ello se requiere no solo que las personas dispongan de la posibilidad de acceso al entorno digital en el hogar, es preciso también contar con ambientes socioculturales y educativos que promuevan explícitamente el dominio del lenguaje digital (Buckingham, 2008), en términos no solo de la capacidad que tengan sino también desde la disposición para involucrarse en entornos que puedan satisfacer sus necesidades y expectativas socioeducativas.

Según Mato (2009), para que las instituciones de educación superior se orienten adecuadamente a un horizonte congruente, deben tomar en consideración los tiempos de acomodo socioeconómico e intercultural y las aspiraciones de los jóvenes y de las comunidades en su amplia pluralidad. En ese sentido, ha sido señalado que la educación intercultural y las tecnologías de la información, como espacios teóricos y prácticos resultan ser mecanismos idóneos para afrontar el desafío de "responder a las exigencias que la globalización y la sociedad de la información impone a los países emergentes en América Latina” (UNESCO, 2013, p.141) pero también para atender la diversidad cultural que habita en los grupos históricamente discriminados (minorías étnicas, culturas juveniles, género, entre otros) y que deben estar inmersos como público sujeto de educación en programas de pregrado y posgrado. 
Es así que a la luz de estos conceptos percibimos que hay aspectos relevantes que no han logrado permear y predominar en las ofertas de posgrado en las últimas décadas, tales como la igualdad de oportunidades, la democratización del conocimiento, y la inclusión de dimensiones claves para el desarrollo integral del ser humano (filosofías y cosmovisiones de los pueblos y nacionalidades). Desde esa mirada buscamos identificar en los programas de posgrados si hay contenidos que actúen en sentido opuesto a la discriminación y que respondan a una perspectiva intercultural y tecnológica. Pero que además por intermedio de sus propuestas, cuestionen y repelen argumentos y prácticas encarnadas e impuestas por un modelo neoliberal dentro y fuera del aula. A partir de ese interés notamos que si bien hay proyectos curriculares que no acuden al lente intercultural, si hay algunos diseñados para atender la diversidad cultural y las necesidades socioeducativas, buscando cambiar el orden del pensamiento educativo tradicional.

Dichas propuestas se perfilan desde tónicas que buscan romper las cadenas hegemónicas, rechazar la opresión, recuperar saberes propios de sus entornos para teorizarlos y combinarlos con los occidentales, y promover una pedagogía encaminada en la formulación de una conciencia crítica (Freire, 1998) para construir sociedades con mejores y mayores oportunidades. En esa línea, los programas sugieren un proyecto transformador que, como lo exaltan Aguado \& Mata (2017), está centrado en las relaciones humanas y en la construcción de una ciudadanía capaz de transitar hacia sociedades más democráticas, justas y sostenibles.

\section{Metodología}

La metodología cualitativa (Marino \& Rodríguez, 2009) resultó apropiada para la exploración y el análisis de los programas de posgrado que buscan adaptar el enfoque intercultural y las TIC en educación, y que en su defecto requieren plantearse desde la construcción teórica. Al contar con escasos estudios que analicen este tipo de propuestas, una metodología de este tipo permite obtener datos con flexibilidad para contarlos e interpretarlos desde sus significancias (Álvarez-Gayou Jurgenson, 2003; Martínez, 1999).

Los dos instrumentos elegidos fueron la herramienta de búsqueda del CES y el análisis documental. La primera posibilitó la revisión de cifras y contenidos de la oferta que compila, regula y avala la máxima instancia en educación superior en Ecuador, y el análisis documental dio paso a revisar la postura teórica en relación con el enfoque intercultural que hemos adoptado, pero también de la propia teoría 
construida desde la narrativa de los programas de posgrado. En palabras de Vickery (1970).

El análisis documental responde a tres necesidades informativas de los usuarios; en primer lugar, conocer lo que otros pares científicos han hecho o están realizando en un campo específico; en segundo lugar, conocer segmentos específicos de información de algún documento en particular; y por último, conocer la totalidad de información relevante que exista sobre un tema específico (p.154).

El uso de esos dos instrumentos lleva a una selección de ideas y categorías y a la obtención de información relevante (Peña \& Pírela, 2007), que por un lado, facilitó la revisión y observación de documentos oficiales (describiendo los programas, sus objetos, contenidos y asignaturas) y por otro, permitió contrastarlos, compararlos y analizarlos desde sus propias construcciones y desde las corrientes teóricas en torno a los enfoques enunciados.

\section{Censo de programas de posgrado en Ecuador: educación y TIC}

En Ecuador, las maestrías se clasifican en dos categorías: de investigación formativa y de investigación académica y científica. Según el Reglamento de Régimen Académico (2018) las de investigación formativa (maestrías profesionales y especializaciones no médicas) son profesionalizantes y profundizan en el conocimiento de la epistemología de la ciencia y desarrollan proyectos investigativos de carácter explicativo o comprensivo con claro aporte al área de conocimiento. Las de investigación académica y científica (maestrías en investigación y doctorados) responden a una labor creativa, sistémica, rigurosa, epistemológica y metodológicamente fundamentada que produce conocimiento susceptible de universalidad, originalmente nuevo y orientado al crecimiento del cuerpo teórico de uno o varios campos científicos.

A decir por la página oficial del CES, el censo de programas de posgrado en Ecuador es amplio. En la Tabla 1 mostramos una delimitación de categorías de programas, tipos y campos amplios de estudio relacionados con educación y TIC. En adelante estas son las mismas categorías que guían la selección de oferta analizada. En ambas dimensiones (maestrías profesionales y de investigación) hay un total de 211 programas, de los cuáles 156 corresponden a educación y 55 a TIC. 
Tabla 1. Categorías y tipos de programas de posgrado en Educación y TIC's en Ecuador

\begin{tabular}{lccc}
\hline \multirow{2}{*}{ CATEGORÍAS } & \multirow{2}{*}{ TIPO* } & \multicolumn{2}{c}{ CAMPO AMPLIO } \\
& & Educación & TIC \\
\hline $\begin{array}{l}\text { Maestrías profesionales y especializa- } \\
\text { ciones no médicas }\end{array}$ & MA + TP & 119 & 42 \\
$\begin{array}{l}\text { Doctorados y maestrías en investiga- } \\
\text { ción }\end{array}$ & MP & 30 & 8 \\
Total & MA + TI & 7 & 5 \\
${ }^{*}$ MA: Maestría Académica; MP: Maestría Profesional; TP: Trayectoria profesional; TI: Trayectoria de investigación \\
\multicolumn{2}{l}{ Fuente: elaboración propia con datos oficiales CES (2020) }
\end{tabular}

\section{Tamaño de la muestra}

El planteamiento parte de una muestra de 62 maestrías, tomadas de la misma fuente oficial del CES en la opción Oferta Vigente del Sistema de Educación Superior del Ecuador. Estos programas están vinculados con las variables "interculturalidad" y "TIC" y se encuentran agrupados en el campo de la educación. Todos están vigentes y corresponden a modalidades de estudios presenciales $(96 \%)$ y de formación en línea y a distancia (4\%).

Figura 1. Oferta de posgrados en Educación con salidas profesionales

Tipo de programa: Maestría Académica con trayectoria profesional

Campo amplio: Educación

60 programas con salida profesional

Fuente: elaboración propia

Figura 2. Oferta de posgrados en Educación con salidas investigativas

Tipo de programa: Doctorados y maestrías de investigación

Campo amplio: Educación

2 programas con salida investigativa

Fuente: elaboración propia 
Tal como se observa en las Figuras 1 y 2, el punto de inicio fue la elección del Tipo de Programa, cuyo rango cuenta con dos opciones: maestrías con trayectoria profesional y de investigación; en este caso se filtró con las dos variables, pues en ambas los criterios responden a los intereses y enfoques del estudio. El segundo paso fue determinar el Campo Amplio, correspondiente a la educación.

\section{Delimitación de la muestra y exposición de resultados}

Valiéndonos de los criterios de las variables, el sistema identificó un total de 62 programas, de los cuales se seleccionaron 18 que incluían en primer lugar, ámbitos concretos de especialización en interculturalidad; en segundo, proponían el enfoque intercultural y el componente tecnológico en sus propuestas curriculares; y tercero, proyectaban tanto una diversificación de conceptos, como una sinergia para abordar la diversidad y sus perspectivas.

Las cuestiones frecuentes que emergieron en esta selección giraron alrededor de tópicos como: diversidad cultural, pedagogías emergentes, virtualidad e innovación educativa; los cuales por sus alcances y configuraciones proponen la incorporación de nuevos conceptos, temas, y visiones en el campo de la educación.

Finalmente, los resultados se categorizan de la siguiente manera y son desarrollados ampliamente en el apartado próximo:

1) maestrías que utilizan la nomenclatura intercultural en sus denominaciones y sus contenidos proponen el enfoque como corpus teórico.

2) maestrías con nociones interculturales en sus descripciones, contenidos, objetos de estudios y asignaturas.

3) maestrías que tienen el componente tecnológico en sus contenidos, descripciones, objetos de estudios y asignaturas.

\section{RESULTADOS}

Este apartado se divide en tres epígrafes: 1) maestrías con nomenclatura intercultural; 2) maestrías con nociones interculturales en sus contenidos, objetos de estudio y asignaturas; y 3) maestrías con contenidos, descripciones y objetos de estudio relacionados con las tecnologías. 


\section{La interculturalidad y las tecnologías en la educación de posgrado}

En la construcción de relaciones sociales más justas y en la búsqueda por alcanzar una educación social y democrática (Aguado \& Mata, 2017), la perspectiva intercultural resulta relevante en el diseño de los programas de posgrado, también llamados de cuarto nivel en Ecuador. A esto se suma que el uso de las tecnologías da lugar a la conformación de escenarios híbridos (Canclini, 2017) globales y glocales (Robertson, 1995) como núcleos de aprendizaje-enseñanza en las nuevas y cambiantes estructuras que propone la sociedad moderna (antes y durante la pandemia del Covid 19). Por consiguiente, conocer la presencia de la interculturalidad y las tecnologías en las alternativas formativas permite adentrarnos en una construcción teórica y práctica al interior de las maestrías y percatarnos cuáles son sus contenidos, cómo operan sus diseños y a que necesidades responden.

\section{Maestrías con nomenclatura intercultural}

En la primera categoría (tabla 2) se constata que actualmente están en vigencia dos programas de posgrado cuya nomenclatura acude al término intercultural. Se trata de las Maestrías en Educación, mención Educación Intercultural, una ofrecida por la Universidad de Cuenca, y la segunda por la Universidad Católica de Cuenca, ambas con sede en el sur ecuatoriano.

Tabla 2. Programas de Maestría en Interculturalidad

\begin{tabular}{lll}
\hline \multicolumn{1}{c}{ Universidad } & $\begin{array}{l}\text { Programa de Maestría } \\
\text { en Interculturalidad }\end{array}$ & \multicolumn{1}{c}{ Asignaturas } \\
\hline Universidad de Cuenca & $\begin{array}{l}\text { Educación, mención } \\
\text { Educación Intercultural }\end{array}$ & $\begin{array}{l}\text { "Pedagogía Intercultural", "Aulas Interculturales", "Cu- } \\
\text { rrículo Intercultural", "Epistemología de la Interculturali- } \\
\text { dad", "Planes y Proyectos Interculturales", }\end{array}$ \\
$\begin{array}{l}\text { Universidad Católica } \\
\text { de Cuenca - Sede Azo- } \\
\text { gues }\end{array}$ & $\begin{array}{l}\text { Educación, mención } \\
\text { "Currículo Intercultural", "Innovación en la Educación In- } \\
\text { tercultural, "Epistemología de la Interculturalidad", } \\
\text { "Marco Normativo de la Interculturalidad". }\end{array}$ \\
\hline
\end{tabular}

Fuente: elaboración propia con datos tomados del CES

En el análisis se puede notar que estos programas centran sus alcances en responder a los problemas de la sociedad actual, atendiendo la diversidad cultural más allá de las dimensiones étnicas y desde un concepto plural para abordar las complejidades epistémicas, socioculturales, didácticas, pedagógicas y tecnológicas. Sus diseños compilan asignaturas que tienen en común el enfoque intercultural, y cuyos nombres replican temas como: aulas interculturales, pedagogía intercultural, 
currículo intercultural, diseño de proyectos y programas, innovación en la educación intercultural y tecnologías emergentes.

Al tratarse de las dos únicas maestrías con esa nomenclatura y características de ese tipo, plantean un escenario aún naciente para la reproducción de los estudios interculturales. Los esfuerzos de las pocas propuestas amparadas en el enfoque intercultural en Ecuador y en países de la región latinoamericana aun resultan insuficientes para hablar de una perspectiva de orden prioritario no solo a nivel discursivo, sino también práctico. Como lo afirma Mato (2009) en el caso de la educación superior, si bien ha habido avances, éstos resultan aun menores que en los otros niveles de formación. No obstante, el educativo es uno de los campos en los cuales, aunque insuficientes, pueden observarse progreso en materia de interculturalidad. Así por ejemplo podríamos citar los programas que llevan adelante la Universidad Veracruzana Intercultural y la Universidad Intercultural de Chiapas en México, o el Programa de Educación Intercultural Bilingüe (PROEIB) en la Universidad Mayor de San Simón en Bolivia.

Es preciso señalar que también hay una especialización en Interculturalidad y Desarrollo, esta es propuesta por la Facultad Latinoamericana de Ciencias Sociales (FLACSO) sede Ecuador y tiene entre sus objetivos fortalecer a actores sociales para que aporten con un enfoque intercultural a sus competencias analíticas, técnicas, teóricas y prácticas. Cabe destacar que la especialización se trata de un programa de posgrado y corresponde a la formación avanzada, en torno a un campo disciplinar o profesional. Requiere una carga horaria entre 1000 y 1040 horas, mientras que las maestrías profesionales estipulan entre 2120 y 2200 horas, y las de investigación desde 2640 hasta 2760 horas (CES, 2020).

\section{Maestrías con nociones interculturales en sus contenidos, objetos de estudios y asignaturas}

Los resultados en la segunda categoría muestran un conjunto de maestrías que tienen en común el campo amplio de la educación y que a manera de nociones entrelazan el enfoque intercultural no de manera protagónica como las anteriores, pero si lo dejan entrever en sus composiciones curriculares.

Las asignaturas reflejan la recuperación disciplinar y sub disciplinar de las Ciencias Sociales y Humanidades en el contexto socioeducativo, y plantean combinaciones conectadas con la sociología, pedagogía, didáctica y antropología. También en este grupo se manifiesta la necesidad de pensar al sujeto no individual sino colectivamente, es decir con las personas e instituciones sociales que lo rodean, pero 
además con los elementos que ayudan a entrelazar y tejer relaciones más justas y simétricas.

Tabla 3. Maestrías en Educación con objetos de estudios y asignaturas asociadas con la interculturalidad

\begin{tabular}{|c|c|c|}
\hline Universidad & Programa de Maestría & Asignaturas con enfoque intercultural \\
\hline $\begin{array}{l}\text { Pontifica Universidad } \\
\text { Católica del Ecuador } \\
\text { Sede Esmeraldas }\end{array}$ & $\begin{array}{l}\text { Educación, mención Inclusión } \\
\text { Educativa y Atención a la Di- } \\
\text { versidad }\end{array}$ & No visibles en la web oficial del CES \\
\hline $\begin{array}{l}\text { UNAE Universidad Na- } \\
\text { cional de Educación }\end{array}$ & $\begin{array}{l}\text { Educación de Jóvenes y } \\
\text { Adultos }\end{array}$ & $\begin{array}{l}\text { "Ética", "Globalización y Cultura", "Socio- } \\
\text { logía y Educación Popular" }\end{array}$ \\
\hline $\begin{array}{l}\text { UTM (Universidad Téc- } \\
\text { nica de Manabí) }\end{array}$ & $\begin{array}{l}\text { Pedagogía. Mención Docen- } \\
\text { cia e Investigación en Educa- } \\
\text { ción }\end{array}$ & $\begin{array}{l}\text { "Realidad Socioeducativa en el Contexto } \\
\text { Ecuatoriano" }\end{array}$ \\
\hline UTM & $\begin{array}{l}\text { Educación, mención Comuni- } \\
\text { cación Educativa }\end{array}$ & $\begin{array}{l}\text { "Cultura en Comunicación Latinoameri- } \\
\text { cana", "Comunicación Lenguaje y Socie- } \\
\text { dad" }\end{array}$ \\
\hline $\begin{array}{l}\text { UIE Universidad Inter- } \\
\text { nacional del Ecuador }\end{array}$ & Psicopedagogía & $\begin{array}{l}\text { "Herramientas Pedagógicas Avanzadas", } \\
\text { "Felicidad como producto interior en la } \\
\text { educación" }\end{array}$ \\
\hline Universidad de Cuenca & $\begin{array}{l}\text { Investigación e Innovación } \\
\text { Educativa }\end{array}$ & $\begin{array}{l}\text { "Educación Intercultural", "Antropología } \\
\text { de la Educación", "Didáctica y Evaluación } \\
\text { para la Diversidad", "Educación y Globali- } \\
\text { zación" }\end{array}$ \\
\hline UTM & $\begin{array}{l}\text { Educación, mención Ense- } \\
\text { ñanza Básica }\end{array}$ & $\begin{array}{l}\text { "Fundamentos Socioculturales de la So- } \\
\text { ciedad y la Familia", "Investigaciones } \\
\text { Educativas y Expresión Artística" }\end{array}$ \\
\hline $\begin{array}{l}\text { UDLA - Universidad de } \\
\text { las Américas }\end{array}$ & Liderazgo Educativo & "Educación inclusiva", "Epedagogy" \\
\hline UTM & $\begin{array}{l}\text { Pedagogía. Mención Bachille- } \\
\text { rato Técnico }\end{array}$ & $\begin{array}{l}\text { "Equidad e Inclusión como Política en la } \\
\text { Educación", "Construcción de saberes" }\end{array}$ \\
\hline $\begin{array}{l}\text { Universidad Nacional de } \\
\text { Loja }\end{array}$ & Educación Básica & "Cultura Andina y prácticas ancestrales" \\
\hline PUCE & Innovación en Educación & $\begin{array}{l}\text { "Interculturalidad e Inclusión", "Práctica y } \\
\text { acción social", "Tic aplicadas a la Educa- } \\
\text { ción" }\end{array}$ \\
\hline
\end{tabular}

Fuente: elaboración propia con datos tomados del CES

Como lo muestra la tabla 3 hay un predominio mayor en cuanto a la inserción de asignaturas sobre inclusión y diversidad. Esto en respuesta, por un lado, a la necesidad de integrar a quienes históricamente son excluidos del sistema educativo por cuestiones de desigualdad y discriminación; y por otro, a incluir lo diverso 
como parte sustancial y enriquecedora de un modelo educativo democrático y participativo, a fin con las políticas y demandas actuales.

En estas arenas observamos que la Universidad de Cuenca oferta una maestría en Investigación e Innovación Educativa cuyo pénsum reúne cuatro asignaturas con enfoque intercultural: "Didáctica y Evaluación para la Diversidad", "Educación Intercultural”, "Educación y Globalización”, y "Antropología de la Educación". La misma institución acoge otro programa de maestría en Antropología de lo Contemporáneo, incluyendo también asignaturas asociadas con la interculturalidad y con la propuesta de estudio del ser humano como protagonista con sus unidades y complejidades (De Soria, 2006).

En esa misma línea hallamos los programas de Innovación y Educación (Pontificia Universidad Católica del Ecuador), Psicopedagogía (Universidad Internacional del Ecuador), Educación con mención en Enseñanza Básica, o Pedagogía (Universidad Técnica de Manabí), que a decir por sus objetos de estudio responden a las demandas contextualizadas del territorio dadas sus características en el marco de los saberes y las bases socioculturales.

También en el master de Psicopedagogía (UIE) encontramos la asignatura "Felicidad como producto interior en la educación" cobrando particular sentido porque hace énfasis a un ámbito poco explorado en los contenidos de los programas a nivel de pregrado y posgrado. Su presencia expone la felicidad como meta primordial para el pleno potencial del ser humano y como objetivo esencial de la educación (Arguís et al., 2012).

Así mismo en esta categoría se pueden distinguir asignaturas cuyas temáticas giran alrededor del trabajo colaborativo, la participación ciudadana, el diálogo de saberes, la interacción social y las emociones. Y que en las arenas de la práctica intercultural resaltan la importancia de un pensamiento transdiciplinar capaz de mostrar lo diverso y complejo como complemento y propuesta de aprendizaje (Paul, 2009).

\section{Maestrías con contenidos, objetos de estudios y asignaturas asociados con tecnologías}

En este apartado constan aquellos programas que derivan de las tecnologías de la información y comunicación aplicadas al campo de la educación. El conjunto de maestrías si bien no incluye en sus nomenclaturas cuestiones relacionadas con la interculturalidad, sus contenidos resaltan la importancia de los elementos tecnológicos en el marco de la diversidad. 
Tabla 4. Programas en Educación con objetos de estudios y asignaturas asociados con TIC

\begin{tabular}{|c|c|c|}
\hline Universidad & Programas de Maestría & Asignaturas con enfoque en las TIC \\
\hline PUCE Ambato & $\begin{array}{l}\text { Pedagogía, mención en } \\
\text { Educación Técnica y } \\
\text { Tecnológica. }\end{array}$ & No visibles en la web oficial del CES \\
\hline Universidad del Pacifico & $\begin{array}{c}\text { Educación, Tecnología e } \\
\text { Innovación }\end{array}$ & $\begin{array}{c}\text { "Multimedia, Tecnología y Aprendizaje", } \\
\text { "Aprendizaje apoyado en Tecnologías", "Mo- } \\
\text { delos de Comunicación Educativa y Tecnoló- } \\
\text { gica" }\end{array}$ \\
\hline $\begin{array}{c}\text { Universidad Técnica del } \\
\text { Norte }\end{array}$ & $\begin{array}{c}\text { Tecnología e Innovación } \\
\text { Educativa }\end{array}$ & $\begin{array}{c}\text { "Diseño y Elaboración de Materiales Didácti- } \\
\text { cos Digitales", "Diseño de Proyectos Educati- } \\
\text { vos con Tic", "Integración Curricular de la } \\
\text { Tecnología Educativa", "Sloodle y Entornos } \\
\text { Educativos 3D", "Software libre aplicado a la } \\
\text { educación" }\end{array}$ \\
\hline $\begin{array}{c}\text { Universidad Católica de } \\
\text { Cuenca }\end{array}$ & $\begin{array}{c}\text { Educación, tecnología e } \\
\text { innovación }\end{array}$ & $\begin{array}{l}\text { "Diseño curricular y Currículo Inclusivo", } \\
\text { "Tecnologías Emergentes para la Innovación } \\
\text { de Procesos Educativos", "Diseño de Recur- } \\
\text { sos Didácticos Innovadores" }\end{array}$ \\
\hline $\begin{array}{l}\text { ECOTEC Universidad } \\
\text { Tecnológica }\end{array}$ & $\begin{array}{c}\text { Tecnología e innovación } \\
\text { educativa }\end{array}$ & $\begin{array}{l}\text { "Diseño Instruccional e Innovación en la Edu- } \\
\text { cación Virtual", "Sistemas Inteligentes en la } \\
\text { Educación", "Plataformas Interactivas", "Desa- } \\
\text { rrollo de cursos en líneas, ubicuidad e integra- } \\
\text { ción de tecnología móvil en la educación" }\end{array}$ \\
\hline $\begin{array}{l}\text { Universidad Técnica } \\
\text { Equinoccial }\end{array}$ & $\begin{array}{l}\text { Educación mención en } \\
\text { Gestión del Aprendizaje } \\
\text { mediado por Tic }\end{array}$ & $\begin{array}{l}\text { "Diseño de Materiales Educativos Digitales", } \\
\text { "Plataformas de Gestión de Entornos Virtua- } \\
\text { les. "Aprendizaje y Entornos Virtuales" }\end{array}$ \\
\hline
\end{tabular}

Fuente: elaboración propia con datos tomados del CES

A diferencia de los programas con enfoque intercultural, los que acuden al componente tecnológico desde el campo de la educación son mayores en cifras. Tal como se puede ver en la tabla 3 , a nivel de posgrado hay un número significativo de programas enmarcados en el campo amplio de la educación y tecnologías.

Por un lado, los objetos de estudio, el perfil de egreso y la descripción de estos revelan la asunción e implementación de ambientes educativos soportados por las tecnologías. La oferta plantea la solución y el abordaje de los desafíos de la virtualidad desde un marco innovador con diferentes dispositivos, estilos y medios de comunicación. A su vez que sugiere un enfoque multi y transdiciplinario, idóneo a permanecer abierto como eje de exploración y conocimiento en constante transformación y descubrimiento. Los elementos que los componen responden a la 
resolución de problemas socioculturales y a la inclusión de más de una disciplina de las ciencias sociales y humanísticas.

En ese sentido, conforme a lo señalado en el proceso de elección de los programas, reiteramos que estos fueron escogidos en función de las variables y contenidos entrelazados con las tecnologías educativas y la innovación educativa. De ese modo detectamos que varios de los programas recogidos en la tabla 4, comparten intereses por el diseño de recursos, de plataformas tecnológicas, de innovación de contenidos y recursos para la implementación de las TIC. Logran concentrar en sus mallas curriculares asignaturas que promueven el pensamiento crítico de la pedagogía, tanto como la democratización y liberación del pensamiento (Dewey, 1995; Freire, 1998), desde el matiz de la modernidad y los cambios innovadores que propone la globalización.

Así, por ejemplo, resaltamos asignaturas como "E-pedagogy", "Tecnologías Emergentes", "Herramientas Colaborativas", "Tecnologías para el Aprendizaje Auto Regulado", "Plataformas Multimedias" y "Aprendizaje en Entornos Virtuales", que en la praxis resultan ser propuestas no solo que van de la mano con los procesos de transformación social y las nuevas matrices de producción y conocimiento, sino que son atractivas e interpeladas entre las generaciones estudiantiles, dadas sus características creativas, innovadoras y flexibles en cuanto acceso, disponibilidad de tiempo y economía a largo plazo.

Las TIC como subrayan Botello \& Rincón (2012) tienen la capacidad de mejorar la comunicación entre los distintos agentes del proceso enseñanza-aprendizaje, en especial entre estudiantes y docentes, lo que incrementa los flujos de información y la colaboración entre ellos, más allá de los límites físicos y académicos de las instituciones de enseñanza. Por lo que cabe destacar que el nivel de conocimiento y experticia del profesorado supone además un reto mayor, pues las propuestas que son visibles en este conjunto de asignaturas refieren una renovación y una capacitación de gran nivel de la planta docente, para afrontar la contienda que propone este paradigma educativo mediado por las tecnologías.

Por último, en las propuestas de este grupo de materias el desarrollo de habilidades y competencias tecnológicas a través de los conocimientos que propone el pensum de estudio, y el incesable interés por desempeñarse en profesiones relacionadas con la digitalización, plantean la necesidad y demanda de una ciudadanía que está incursionando y haciendo uso de las herramientas TIC para hacer frente a los retos y desafíos que propone la globalización, y que ve en las salidas profesionales no solo el mejoramiento de sus capacidades profesionales sino la posibilidad 
de incursionar en una de las profesiones con mayor impacto en el mercado laboral. Eso ha conllevado a que en los últimos años la presencia no sólo de asignaturas relacionadas a las tecnologías de la enseñanza, sino que los programas que se oferten a nivel de pregrado y posgrados adquieran mayor interés entre la población ecuatoriana.

\section{DISCUSIÓN}

\section{El papel que ocupa la interculturalidad y las TIC en la formación de posgrado}

\section{Lo intercultural}

En el panorama analizado, observamos que la interculturidad no es una propuesta integrada significativamente como lo determina la normativa constitucional ecuatoriana con sus principios y fines garantistas para una educación intercultural. Puesto que, pese a que la ley contempla que su enfoque debe ser transversal y obligatorio, este ha logrado calar únicamente desde un plano discursivo.

El instrumento legal ecuatoriano sobre el que se articula la política pública y el estado de democracia, apunta la necesidad de:

Desarrollar, fortalecer y potenciar el sistema de educación intercultural bilingüe, con criterios de calidad, desde la estimulación temprana hasta el nivel superior, conforme a la diversidad cultural, para el cuidado y preservación de las identidades en consonancia con sus metodologías de enseñanza y aprendizaje (Art. 57 Constitución de la República del Ecuador, 2008).

Esa misma línea también la Ley Orgánica de Educación Superior (LOES) y la Ley Organiza de Educación Intercultural (LOEI), esta última garantista del derecho a la educación en el marco de la valoración de la diversidad cultural como política para el Buen Vivir ${ }^{1}$, articulan sus políticas, alcances y responsabilidades teniendo como base la interculturalidad y su propósito de construir una ciudadanía que valore la diversidad y fortalezca lazos de unión para convivir armónicamente en medio del respeto, la diferencia y la colaboración mutua.

No obstante, notamos que aún hay disonancias en el terreno práctico, dado que lo intercultural se presenta con frecuencia como un adjetivo con el que se

\footnotetext{
${ }^{1}$ El Buen Vivir es un principio constitucional en el Ecuador que recoge una visión del mundo centrada en el ser humano, como parte de un entorno natural y social (Mineduc, 2020). Se trata de un eje esencial de la educación porque contempla la preparación de futuros ciudadanos con valores y conocimiento para fomentar el desarrollo del país.
} 
califica a educadores, escuelas, programas educativos o recursos. Los vacíos y grietas que proyectan la desigualdad expresan la urgencia de trabajar desde un proyecto y espacio en común, que puede ser la academia, desde donde germinen propuestas, políticas y cambios transformadores para hacer posible la interculturalidad y vivirla desde el enriquecimiento recíproco.

La interculturalidad como eje transversal en la vida cotidiana y en el mundo académico supone un reto social, político pero sobre todo ético y reflexivo, porque como lo afirma Guerrero (2008) debemos iniciar reconociendo más críticamente que la colonialidad del poder, del saber y del ser, no son exterioridades presentes en los otros, o que solo se expresan en el poder, sino que están muy presentes en cada uno de los ciudadanos, atravesando sus pensamientos e imaginarios, subjetividades, cuerpos y prácticas diarias. $\mathrm{Y}$ es por tanto, un trabajo introspectivo, el punto de partida para deslegitimar prácticas desiguales y excluyentes.

\section{Lo tecnológico}

Desde el punto de vista de Salazar (2005) si nos referimos a la tecnología en el campo educativo los conceptos de globalización y glocalización resultan imprescindibles para el análisis. Ese abordaje permite comprender las múltiples interconexiones entre lo global y lo local, y a fijarnos en cómo se tejen las relaciones dentro y fuera de la escuela, así como las maneras cómo interactúan las personas con procesos generacionales, necesidades y preferencias distintas, en medio del uso de un dispositivo capaz de conectar el ser humano con el conocimiento que deviene de la escuela.

Al respecto Hall \& Du Gay (2006) afirman que con la implementación de dispositivos innovadores se puede incursionar y construir identidades más plurales y diversas, menos estancadas y mayormente unificadas. Se da cabida, así, a términos que no son disonantes a la interculturalidad y a las tecnologías, y que se recrean como nuevos dispositivos de enseñanza y aprendizaje, no integrados antes en propuestas similares. En ese sentido, nos referimos a cuestiones que abordan la comunicación educativa y tecnológica, el diseño y elaboración de materiales digitales y educativos, la ubicuidad y movilidad tecnológica educativa, la integración curricular tecnológica y también la creación de entornos educativos 3D.

Para la sociedad la puesta en marcha de mecanismos que ayudan a mejorar el acceso a la educación desde las tecnologías representa hacer uso eficiente, flexible y práctico de las herramientas que subyacen de las tendencias y metodologías 
centradas en el entorno digital, y que por tanto, deben estar presente en la propuesta formativa en todos los niveles educativos.

A diferencia del enfoque intercultural, el análisis permite evidenciar que en cuanto a las tecnologías si hay una mayor disposición por insertar nuevos dispositivos de aprendizaje y explorar campos que pueden mejorar los procesos de enseñanza y aprendizaje. Las propuestas formativas de maestrías muestran avances no solo en la preparación y formación de la planta docente, sino también en la implementación de la infraestructura tecnológica, tanto como en el acompañamiento que se brinda a docentes y estudiantes para garantizar resultados exitosos.

Esto resulta importante, porque como lo aseguran Alderete \& Formichella (2016) la interrelación de varios factores, entre ellos, el grado de confidencia, la familiaridad de los docentes, estudiantes, madres y padres con las TIC, el acceso a los recursos en el hogar, la escuela o cualquier otro entorno es clave para gestionar los entornos virtuales. Contrariamente, cuando esto no ocurre, la exclusión educativa propicia, al igual que en entornos de aprendizaje presenciales, nuevas formas de segregación y desigualdades por cuestiones tecnológicas.

Finalmente, vale mencionar que en los programas repasados desde la dimensión intercultural se integra el componente tecnológico. Lo mismo ocurre con algunos programas que desde las tecnologías recuperan nociones socioculturales, logrando hibridarlas y flexibilizarlas bajo una perspectiva humanista. Así, por ejemplo, el programa ofertado por la Universidad Tecnológica Equinoccial, en Educación con mención en Gestión del Aprendizaje mediado por Tic, desde su objeto de estudio, propone contribuir a lograr las aspiraciones desde lo social y cultural, buscando potenciar el reconocimiento, el respeto y la promoción de la diversidad cultural, la interculturalidad y la inclusión social, desde el uso de las TIC.

\section{El presente y el futuro de la interculturalidad y las tecnologías}

Pese a que la incorporación del enfoque intercultural y las TIC sigue siendo paulatino, y que su panorama actual no es el ideal, también hay luces que dan cuenta de un presente que está cambiando y de un futuro prometedor si se logra la participación de la sociedad en su conjunto en un proyecto común, reciproco y de respeto mutuo. Por ello si hablamos de una proceso intercultural e integrador de las nuevas tecnologías para el aprendizaje en la vida dentro y fuera de la academia, se debe asumir prioritariamente la participación de los movimientos sociales, las comunidades, pueblos y nacionalidades indígenas, la sociedad en general, y por ende sus costumbres, necesidades y formas de mirar el mundo que los rodea. 
Los estudios en este campo apuntan a que las formas para integrar lo intercultural requiere de revisiones de modelos y sistemas educativos, establecer un diálogo intercultural, es decir un camino más cercano, pues este representa un proyecto articulador, una respuesta alternativa que se opone a la asimilación o aceptación de la alteridad en una sociedad monoculturalmente predeterminada (FornetBetancourt, 2001). Es decir, de un proyecto que acoja la diversidad educativa (docentes, estudiantes, comunidad educativa, estado, sociedad) y proponga soluciones y mecanismos de enseñanza-aprendizaje consensuadamente. De ahí que el rol que tienen las universidades y sus propuestas formativas de posgrado junto a los diseños de políticas, programas y proyectos del estado, deben apostar por abordar la interculturalidad y las tecnologías de la información como ejes transversales en los diversos escenarios y realidades geográficas, históricas, sociales y culturales presentes en el país.

La educación del presente invita a construir desde las humanidades y las ciencias sociales un conocimiento transdiciplinar que sea capaz de organizar los saberes que trascienden las disciplinas de una forma radical, ampliándolos y cohesionándolos hacia otros con un propósito común (Morín, 1999) y en beneficio de todos. Como señala Fernández (2012), estamos en un momento que demanda la recomposición de las relaciones sociales, en el que se habla de algo completamente nuevo, o desconocido, pero que tiene sus raíces en la civilización humana, y que de cualquier manera se trata de un fenómeno del que ahora más que nunca estamos tomando consciencia y al cual los sistemas estatales de educación deben atender.

Tanto en el presente como en el futuro las decisiones y la integración de actitudes positivas con las que se contemple la diversidad cultural y las competencias comunicativas, permitirán a decir por Aguado (2003) tener la capacidad de manejar conflictos en situaciones diversas, tener conciencia sobre la propia cultura y visibilizar como ésta influye en la visión e interpretación de la realidad.

\section{CONCLUSIONES}

El objetivo de visibilizar la puesta en valor del enfoque intercultural y de las TIC en la oferta de posgrado en educación fue cumplido en la medida que se identificó el grado de implicación que tienen dichas categorías y como operan para solventar los dilemas y los problemas reales que enfrenta la población.

La exacerbación de una realidad dispar, desigual que está presente en Ecuador y América latina ha impulsado el surgimiento de políticas públicas incidentes en el plano socioeducativo para reconocer la diversidad como una fuente 
inagotable de conocimientos y para devolver los derechos universales que tienen las personas de aprender en otros contextos. En tal sentido, los avances presentados en materia educativa y los instrumentos constitucionales fomentan la construcción de un estado y de una educación a nivel de maestrías, dinámica, inclusiva e intercultural.

Y aunque el escenario no es el ideal porque no podemos hablar de un estado ni de un modelo educativo íntegramente intercultural, hemos visto como los programas desde la necesidad de ampliar su visión de futuro y de liderar cambios estructurales en la sociedad, están incorporando elementos y enfoques transversales en los diseños y salidas profesionales, haciendo frente a las complejidades que propone la diversidad. Al respecto, en lugar de obviar lo diferente, incluyen y expanden sus horizontes hacia espacios y personas que trascienden el entorno educativo para interpretar y convivir en un mundo pluralmente diverso y empático.

Los contenidos que acogen las maestrías cruzan por temas sensibles a nivel colectivo que superan conceptos tradicionales en torno a lo étnico, pues evidencian una perspectiva global que involucra planteamientos concretos alrededor de las clases sociales, artes, nuevas formas de relacionamiento, dispositivos 3D, inteligencias educativas, espacios interactivos multimedias, entre otros. En ese plano las humanidades cobran un papel significativo al traer enfoques y reflexiones críticas muy conectadas con la esencia y la sensibilidad del ser humano. De ahí que podamos decir que la manera en la que se miran y se tratan las cuestiones interculturales y tecnológicas no pierden de vista al ser humano como principal protagonista del que hacer educativo y social. Dicho eso, resulta necesario considerar que la educación desde una noción general, puede ser intercultural, porque reconoce la diversidad como característica humana (Aguado, 2003) y su naturaleza se entrelaza con diferentes dimensiones y estructuras.

En ese mismo orden, la puesta en valor, el uso y apertura de unos dispositivos socioculturales y digitales contribuyen a tener una educación socialmente más justa, participativa y equitativa. Estos proporcionan herramientas en el ejercicio de una ciudadanía intercultural, para que no haya ciudadanos de primera o segunda, sino que se arbitren los procedimientos para que todos sean tratados iguales (Cortina, 2006) dentro y fuera del sistema escolar y con las mismas oportunidades de acceso y permanencia en las instituciones.

En tal sentido, la interculturalidad y las TIC además de conceptos son prácticas colectivas y proyectos sociales que pueden crear mejores oportunidades teniendo de base la diversidad cultural y la innovación. Y que por tanto las políticas 
y agendas en torno a estos temas no pueden funcionar aisladamente de otros actores como la academia y la ciudadanía, pues se requiere de la intervención y participación social en su conjunto para trabajar por un proyecto en común que cimente las bases de una sociedad intercultural. De ese modo la academia desde su papel social debe repensar la universidad como un espacio en permanente construcción y transformación, en el que se forman los ciudadanos interculturales.

Finalmente, aunque es inexcusable desarrollar más estudios empíricos sobre los resultados de prácticas socioculturales en todo el sistema educativo, conviene seguir explorando especialmente los programas ofertados a nivel de posgrado en este campo de estudios. Pues de una mano, permiten conocer el impacto y la repercusión que tienen a nivel social como propuesta de cambio, y por otra, conocer el grado de aceptación de la población y su involucramiento con propuestas que ayuden a solucionar los problemas que les impiden mejorar sus condiciones de vida.

\section{REFERENCIAS}

Adam, T. (2007). Social housing reform in the transatlantic world before World War I, ponencia presentada en el taller Private Wealth, Public Welfare: Philanthropy and Social Knowledge in a Transatlantic World. Rockefeller Archive Center.

Álvarez-Gayou Jurgenson, J. L. (2003). Cómo hacer investigación cualitativa: Fundamentos y metodología. Paidós.

Aguado, T. (2003). Pedagogía Intercultural. Editorial UNED.

Aguado, T., \& Mata, P. (2017). Educación intercultural. Editorial UNED.

Alderete, M., \& Formichella, M. (2017). El acceso a las TIC en el hogar y en la escuela: su impacto sobre los logros educativos. Revista de economía del rosario, 19(2), 221-242.

Arguís, R., Bolsas, A. P., Hernández, S., \& Salvador, M. M. (2012). Programa "aulas felices". Universidad de Zaragoza.

Botello, A, \& Rincón, G. (2014). La influencia de las TIC en el desempeño académico de los estudiantes en América Latina: Evidencia de la prueba Pisa 2012. Memorias Virtual Educa, Memorias VE2014.

Buckingham, D. (2008). Más allá de la tecnología. Aprendizaje infantil en la era de la cultura digital. Manantial.

Canclini, N. (2017) Culturas híbridas, poderes oblicuos. De Bolsillo.

Carrillo, A. T., \& Becerra, A. J. (2004). La construcción del objeto y los referentes teóricos en la investigación social. La práctica investigativa en ciencias sociales, (13), 13-26.,

Charle, C; Vincent, J. \& Winter, J (eds.) (2007). Anglo-French attitudes: comparisons and transfers between English and French intellectuals since the eighteenth century. Manchester University Press.

Cortina, A. (2002). Ciudadanía intercultural. Glosario para una sociedad intercultural. Philosopbica, (27) 7-15.

De Soria, A. (2006). Antropología de la educación para la formación de profesores. Educación y educadores, 9(2), 149-167. 
Dewey, J. (1995). Democracia y educación: una introducción a la filosofía de la educación. Ediciones Morata.

Dietz, G. (2017). Interculturalidad: una aproximación antropológica. Perfiles educativos, 39(156), 192-207.

Fernández, S. (2002). La glocalización de la comunicación. Ámbitos. Revista Internacional de Comunicación, (7-8), 151-163.

Freire, P. (1998). Pedagogía de la liberación. Editora Moraes.

(1970). Pedagogía del oprimido. Siglo veintiuno editores, SA.

Fornet-Betancourt, R. (2001). Transformación intercultural de la filosofía: ejercicios teóricos y prácticos de filosofía intercultural desde Latinoamérica en el contexto de la globalización (Vol. 11). Desclée de Brouwer.

García, F. \& Granados, J. (1999). Lecturas para educación intercultural. Ediciones Trotta.

Guerrero, P. (2008). Interculturalidad, nacionalidades indias, universidad y procesos políticos en Ecuador. Cuadernos Interculturales, 6(10), 159-169.

Leiva, J. \& Moreno, N. (2011). Construyendo comunidades virtuales de aprendizaje intercultural en la escuela. Actas del VI Encuentro "Tendiendo puentes hacia la interculturalidad". Universidad de Málaga.

Asamblea Nacional (2015). Ley Orgánica de Educación Intercultural. Gobierno de Ecuador.

Asamblea Nacional (2008). Constitución de la República del Ecuador. Ecuador.

Marino, T., \& Rodríguez, M. (2009). Un estudio exploratorio sobre heurísticas en estudiantes de un curso de matemática de nivel pre-universitario. Paradigma, 30(2), 159-178.

Martínez, M. (2006). La investigación cualitativa (síntesis conceptual). Revista de investigación en psicología, 9(1), 123-146.

Mateos, S., \& Dietz, G. (2014). Resignificaciones locales de los discursos transnacionales de educación superior intercultural en Veracruz. Revista mexicana de investigación educativa, 19(60), 45-71.

Mato, D. (2009). Instituciones interculturales de educación superior en América Latina. Procesos de construcción, logros, innovaciones y desafíos. IESALC.

Morín, E. (1999). Introducción al pensamiento complejo. Editorial Gedisa

Hall, S., \& Du Gay, P. (Eds.). (2006). Questions of cultural identity. Crane Resource Centre. (2) pp-273.

Hepp, P. (2009). Las tecnologías digitales en contextos interculturales. CUHSO· Cultura-HombreSociedad, 17(1), 83-90.

Paul, P. (2009). Formação do sujeito e transdisciplinaridade: história de vida profissional e imaginal. TRIOM.

Peña, T., \& Pirela, J. (2007). La complejidad del análisis documental. Información, cultura y sociedad, $16,55-81$.

Priegue, D., \& Leiva, J. (2012). Las competencias interculturales en la sociedad del conocimiento: reflexiones y análisis pedagógico. Edutec. Revista Electrónica De Tecnología Educativa, (40), a207.

RRA Régimen Académico, R. (2019). Reglamento de régimen académico. Ecuador: Asamblea Nacional del Ecuador.

Robertson, R. (1995). Glocalization: Time-space and homogeneity-heterogeneity. Global modernities, 2(1), 25-44.

Salazar, N. (2005). Más allá de la globalización. La política y sociedad, 42(1), 135-149. 
Sánchez, M. (2020). Estudios interculturales desde la educación. Revista historia de la educación latinoamericana, 22(34), 8-16.

Savater, F. (2001). El valor de educar. Educere, 5(13), 93-102.

Superior, C. D. E. (2014). Consejo de Educación Superior. Gobierno de Ecuador.

Tipa, J. (2018). ¿De qué me sirve la interculturalidad? Evaluación de la Universidad Intercultural de Chiapas por sus estudiantes. ALTERIDAD. Revista de Educación, 13(1), 56-71.

UNESCO, (2013), Situación Educativa de América Latina y el Caribe: Hacia la educación de calidad para todos al 2015.UNESCO Santiago de Chile.

(2005). Proyecto de Plan de Aplicación Internacional del Decenio de las Naciones Unidas de la Educación para el Desarrollo Sostenible. Doc. 171 ex/7.UNESCO.

Vickery, B. (1970). Techniques of information retrieval. Editorial Butterworths

Walsh, C. (2005). Introducción. ¿Qué es la interculturalidad y cuál es su significado e importancia en el proceso educativo? En La interculturalidad en educación (pp.4-19) Ministerio de Educación del Perú - UNICEF. LIBRO UNICEF2.cdr (cultura.pe)

Zambrano, F \& Sant' Ana, H. (2020). Academia, Estado y Activismo Femenino. Antropología Experimental, (20), 73-82.

\section{FANNY TUBAY ZAMBRANO}

Profesora investigadora de la Universidad de Cuenca, adscrita al Departamento de Estudios Interculturales de la Facultad de Filosofía. Graduada del Máster Euro Latinoamericano en Educación Intercultural y Doctorada en Educación en la línea de especialización en Estudios Interculturales en Educación (UNED España). Ámbitos de investigación en estudios interculturales y género, racismo en la educación, diálogo de saberes.

fannym.tubay@ucuenca.edu.ec fannytubay@hotmail.com https://orcid.org/0000-0002-9156-0956

Tubay Zambrano, F. (2021). Interculturalidad y TIC en los posgrados de educación en el Ecuador. Bellaterra Journal of Teaching \& Learning Language \& Literature, 14(3), e937.

https://doi.org/10.5565/rev/jtl3.937

Rebut / Recibido / Received / Reçu: 19-08-2020

Acceptat / Aceptado / Accepted / Accepté: 12-09-2021

https://revistes.uab.cat/jtl3/ 